\title{
Angiopoietin-1 variant reduces LPS-induced microvascular dysfunction in a murine model of sepsis
}

\author{
Alessio Alfieri ${ }^{1 *}$, Jay J Watson ${ }^{1}$, Richard A Kammerer ${ }^{2}$, Mohammed Tasab ${ }^{3}$ Pavlos Progias ${ }^{3}$, Kimberly Reeves ${ }^{1}$, \\ Nicola J Brown ${ }^{1+}$ and Zoe L Brookes ${ }^{1+}$
}

\begin{abstract}
Introduction: Severe sepsis is characterised by intravascular or extravascular infection with microbial agents, systemic inflammation and microcirculatory dysfunction, leading to tissue damage, organ failure and death. The growth factor angiopoietin (Ang-1) has therapeutic potential but recombinant Ang-1 tends to aggregate and has a short half-life in vivo. This study aimed to investigate the acute effects of the more stable Ang-1 variant matrilin-1angiopoietin-1 (MAT.Ang-1) on the function of the microcirculation in an experimental model of sepsis, and whether any protection by MAT-Ang-1 was associated with modulation of inflammatory cytokines, angiogenic factors or the endothelial nitric oxide synthase (eNOS)-Akt and vascular endothelial (VE)-cadherin pathways.
\end{abstract}

Methods: Aluminium window chambers were implanted into the dorsal skinfold of male $\mathrm{C} 3 \mathrm{H} / \mathrm{HeN}$ mice (7 to 10 weeks old) to expose the striated muscle microcirculation. Endotoxemia was induced by intraperitoneal injection of lipopolysaccharide (LPS, $1 \mathrm{mg} / \mathrm{kg}$ at 0 and 19 hours). MAT.Ang-1 was administered intravenously 20 hours after the onset of sepsis. Microcirculatory function was evaluated by intravital microscopy and Doppler fluximetry.

Results: Endotoxemia resulted in macromolecular leak, which was ameliorated by MAT.Ang-1 post-treatment. LPS induced a dramatic reduction in tissue perfusion, which was improved by MAT.Ang-1. Proteome profiler array analysis of skeletal muscle also demonstrated increased inflammatory and reduced angiogenic factors during endotoxemia. MAT.Ang- 1 post-treatment reduced the level of IL-1 $\beta$ but did not significantly induce the expression of angiogenic factors. MAT.Ang-1 alone did not induce leak or increase angiogenic factors but did reduce vascular endothelial growth factor expression in controls.

Conclusion: Administration of MAT.Ang-1 after the onset of sepsis protects the microcirculation from endotoxemia-induced vascular dysfunction through reducing inflammation but without pro-angiogenic actions, thus representing a novel, potential pharmacotherapeutic agent for the treatment of sepsis.

\section{Introduction}

Sepsis results from the presence of systemic or localised infections, initiating devastating proinflammatory effects on the microcirculation [1-3]. These effects lead to increased microvascular permeability and leukocyte activation, along with reduced vascular resistance and blood flow [4], and without treatment they may result in tissue damage, multiple organ failure and death. Severe sepsis

\footnotetext{
* Correspondence: alessio.alfieri@kcl.ac.uk

† Contributed equally

'Microcirculation Research Group, Faculty of Medicine, Dentistry and Health, University of Sheffield, Sheffield S10 2RX, UK

Full list of author information is available at the end of the article
}

has a mortality rate of approximately $30 \%$ in the USA [5], which represents the second largest killer after cardiovascular disorders. Corticosteroids and drotrecogin-alfa have been recommended for use in some circumstances [6], but effective therapeutic options remain scarce and basic treatment still revolves around optimising fluid resuscitation and antibiotic therapy. In addition, these therapies may improve tissue perfusion but do not reverse active inflammatory processes, thus there is currently a great need for novel therapeutic strategies for severe sepsis.

Angiopoietin (Ang-1) is an oligomeric-secreted glycoprotein that comprises, together with Ang-2, Ang-3 and
C Biomed Central

() 2012 Alfieri et al.; licensee BioMed Central Ltd. This is an open access article distributed under the terms of the Creative Commons Attribution License (http://creativecommons.org/licenses/by/2.0), which permits unrestricted use, distribution, and reproduction in any medium, provided the original work is properly cited. 
Ang-4, a vascular-specific family of growth factors [7]. These ligands bind to the Tie- 2 receptor, which is expressed on the vascular endothelium. Ang-1 is essential during angiogenesis, being required for correct organisation and maturation of newly formed vessels [7]. However, it also maintains the structural integrity of the mature vasculature [8], regulating microvascular permeability via several mechanisms, including: (i) Ang-1bound Tie-2 signalling through Akt to endothelial nitric oxide synthase (eNOS), promoting quiescence and survival; (ii) sequestration of non-receptor tyrosine kinase $\mathrm{Src}$ through the RhoA downstream target $\mathrm{mDia}$, which prevents Src-mediated vascular endothelial (VE)-cadherin internalisation, thus stabilising inter-endothelial junctions [8]. Being part of the intracellular pathway for nitric oxide (NO)-mediated vasodilation, eNOS also modulates blood flow and tissue perfusion [9].

Use of Ang-1 has proven beneficial in murine models of endotoxemia, as adenoviral-delivered Ang-1 improved survival rates from $60 \%$ to $91 \% 60$ hours following lipopolysaccharide (LPS) administration, correlating with reduced lung oedema and injury [10]. More recently, human recombinant Ang-1, administered 8 hours before and in combination with LPS, also protected mice against pulmonary hyperpermeability in vivo through a p190 RhoGAP-dependent mechanism [11]. However, adenoviral delivery is not plausible in humans, while a largescale production of recombinant Ang-1, which has a short half-life in vivo [12], is hindered by the aggregation and insolubility of this protein [13]. Moreover, septic patients need efficacious treatments after the disease has already developed [6].

With Ang-1 demonstrating exciting therapeutic potential during sepsis, we considered it important to find alternatives that acted via the Tie- 2 pathway with potential for translation into human medicine. Matrilin-1angiopoietin-1 (MAT.Ang-1) is one such compound, being more soluble (>95\% vs. 60 to $70 \%$ ) than the native Ang-1. We produced this Ang-1 variant by replacing the central coiled-coil domain and N-terminal domain of Ang-1 with the short coiled-coil domain of human matrilin-1 [14]. In our study, we used MAT. Ang-1 rather than the more well-known variant COMP. Ang-1, as MAT.Ang1 has comparable activity with wildtype Ang-1, similarly forming a mixture of tetramer and trimer units [13]. The comparable activity between Ang1 and MAT.Ang-1 also suggests that these basic units are active species.

This study investigated the protective effects against sepsis by MAT.Ang-1 in vivo, focusing on changes in microvascular permeability, resistance and blood flow. Importantly, we used a therapeutic protocol where MAT-Ang-1 was administered 20 hours after the onset of sepsis. We then determined whether any acute beneficial effects by MAT-Ang-1 were associated with concurrent modulation of inflammatory or angiogenesis factor expression, and the eNOS-Akt or VE-cadherin signalling pathways.

\section{Materials and methods \\ Animals}

Male $\mathrm{C} 3 \mathrm{H} / \mathrm{HeN}$ mice ( 7 to 10 weeks old; $n=24$ ) were obtained from Charles River (Margate, Kent; UK). All procedures were performed in compliance with the UK Home Office Animal Scientific Procedures Act (1986), under $\mathrm{HO}$ project licence number 40/2972, with rigorous ethical and statistical review by both the Home Office and the University of Sheffield. Investigations conformed to the Guide for the Care and Use of Laboratory Animals published by the US National Institutes of Health $(\mathrm{NIH}$ Publication No. 85-23, revised 1996; Assurance No. A5463-01 for the University of Sheffield).

\section{MAT.Ang-1 production}

The fibrinogen-like domain of human Ang1 (UniProt Q15389, residues 266 to 498), fused at its $\mathrm{N}$ terminus to the coiled-coil domain of human matrillin-1 (UniProt P21941, residues 445 to 496, Mat1-Ang1), was amplified by PCR and cloned into a modified pCEP-Pu vector that contained a secretory signal sequence for BM-40/osteonectin [15]. The recombinant protein was obtained by expression in HEK293 EBNA cells using Lipofectamine according to the manufacturer's instructions (Invitrogen; Paisley, UK). After selection with puromycin $(5 \mu \mathrm{g} / \mathrm{ml})$, cells were expanded for the production of the recombinant protein. Serum-free supernatants were harvested from transfected cells, and the recombinant protein was purified by immobilised metal affinity chromatography on $\mathrm{Ni}^{2+}$-Sepharose according to the manufacturer's instructions (Amersham Biosciences; Little Chalfont, Buckinghamshire; UK), dialysed against PBS and stored at $-80^{\circ} \mathrm{C}$ for further use.

\section{Dorsal microcirculatory chamber}

Mice were anaesthetised with hypnorm and diazepam $(1: 1,0.1 \mathrm{ml} / 100 \mathrm{~g}$ intraperitoneally (i.p.)) and aluminium frames were surgically implanted onto the dorsal skinfold of animals. Briefly, on the left side a circular area of dermis and subcutis was surgically removed and the single layer of exposed striated muscle was covered by a glass window (8 mm diameter) [16]. All chambers were devoid of air bubbles, infection or vascular thrombosis.

\section{Intravital microscopy and experimental groups}

Observation of the dorsal microcirculatory chamber was performed using intravital microscopy 3 days after surgery, during sedation with ketamine and xylazine (10 and $1 \mathrm{mg} / \mathrm{kg}$ i.p.). 
Animals were placed on the stage of a modified Leica microscope (307-072.057; Leica; Milton Keynes, Buckinghamshire; UK), equipped with a tungsten lamp for transmitted light and a mercury lamp for fluorescent light microscopy (excitation filter 460 to $490 \mathrm{~nm}$ ), and preparations were observed through a $10 \times$ objective $(0.3$ numerical aperture; Nikon; Kingston Upon Thames, Surrey; UK). Digital images were captured using a CCD camera (TK-C1360B; JVC; London, UK), displayed on a high-resolution monitor (PVM-1443; Sony; Thatcham, Berkshire; UK) and recorded by video data recorder (VDR-3000; Holdan; Hadfield, Glossop; UK) onto DVD disks (Verbatim 16× DVD+R; Verbatim; Egham, Surrey; UK) for later off-line analysis. The preparations were briefly scanned with low-level transmitted light for the study of the skeletal muscle microcirculation. Two areas of interest were identified - each containing main feeding arterioles (50 to $100 \mu \mathrm{m}$ ) and venules (100 to $250 \mu \mathrm{m}$ ), pre-capillary arterioles (20 to $30 \mu \mathrm{m}$ ) and post-capillary venules ( 30 to $45 \mu \mathrm{m}$ ) - and were recorded for 30 seconds using epi-illumination every hour between 20 and 24 hours.

Fluorescein isothiocyanate was bound to bovine serum albumin [17] and administered into the tail vein at 20 hours of the experimental protocol (66 kDa FITC-BSA, $200 \mu \mathrm{l} / 100 \mathrm{~g}$ intravenously; Sigma; Gillingham, Dorset; UK) to allow observation of the microcirculation. Animals were allocated into four experimental groups: control $(n=6), 1 \mathrm{mg} / \mathrm{ml}$ saline i.p. repeated at 0 and 19 hours; LPS $(n=6)$, B55:055, 600,000 endotoxin units per $\mathrm{mg}$ (Sigma), $1 \mathrm{mg} / \mathrm{ml}$ i.p. at 0 and 19 hours; LPS + MAT.Ang- $1(n=6), 1 \mathrm{mg} / \mathrm{ml}$ LPS i.p. at 0 and 19 hours $+33 \mu \mathrm{g}$ intravenously MAT.Ang-1 at 20 hours; and MAT.Ang- $1(n=6), 33 \mu \mathrm{g}$ intravenously at 20 hours $[18,19]$.

\section{Image analysis: macromolecular leak and vessel diameter} The image analysis software Image-Pro Plus (version 6.0; Media Cybernetics; Rockville, MD; USA) was used off-line on fluorescent images obtained between 20 and 24 hours to measure interstitial fluorescence of FITC-BSA (macromolecular leak) adjacent to post-capillary venules [20]. Image-Pro Plus software assigned an integer value to the brightness of fluorescence, using an arbitrary 8-bit greyscale (range 0 to 255), at three distinct interstitial areas $\left(900 \mu \mathrm{m}^{2}\right)$ immediately adjacent $(<2 \mathrm{~mm})$ to a randomly selected post-capillary venule. This provided a mean value for the leakage from one post-capillary venule per each animal at every time point.

To determine diameters of arterioles and venules, Image-Pro Plus was calibrated with a micrometer specifically designed for the camera and monitor. Three lines were drawn across the vessels (including the lumen and vessel wall) to obtain a median value (in micrometres), and the diameter of one randomly selected feeding arteriole, one feeding venule, one pre-capillary arteriole and one post-capillary venule were measured in each animal at every time point.

\section{Laser Doppler fluximetry: blood flow}

At the end of the microscopic evaluation (24 hours of the experimental protocol), tissues in the chambers were scanned using a MoorLDI2 V5.x Doppler imager (Moor Instruments; Axminster, Devon; UK). This instrument used a single $2 \mathrm{~mW}, 632.8 \mathrm{~nm}$ visible red helium neon laser and was positioned $20 \mathrm{~cm}$ from the window chamber and scanned with a raster pattern over a $5 \mathrm{~cm} \times 5 \mathrm{~cm}$ area inclusive of the microcirculation and surrounding tissue, with a spatial resolution of $256 \times 256$ pixels and a scan rate of 4 milliseconds/pixel. The laser beam was positioned perpendicular to the chamber and generated two-dimensional colour-coded images of blood flow with corresponding photo-images based on light intensity. Images were stored on a compatible laptop computer. Perfusion images were analysed using the MoorLDI dedicated image analysis software (version 3.0). A square region of interest that included the main branch of the microcirculatory network was outlined on each image and used to calculate the area-averaged flux. All individual measurement values represent a mean flux from the measured region of interest in perfusion units.

\section{Cytokine and angiogenesis profiling}

Twenty-four hours after LPS administration, abdominal muscle was removed and snap frozen. Tissues were homogenised in a lysis buffer containing PBS, Triton X-100 1\% and protease inhibitor cocktail (Roche; Burgess Hill, West Sussex; UK), and their protein concentration was measured by the Bradford assay using BSA as standard. Protein expression of cytokines/chemokines and angiogenic factors was determined by two different multiplex arrays for simultaneous detection (R\&D Systems; Abingdon, Oxfordshire; UK) according to the manufacturer's protocol. A $200 \mu \mathrm{g}$ sample of tissue proteins was used for this array.

\section{Immunoblotting}

Proteins ( 20 or $80 \mu \mathrm{g}$; obtained as described above) were boiled (10 minutes) and loaded on 12\% SDS-PAGE and transferred onto nitrocellulose membranes for 45 minutes at $250 \mathrm{~mA}$. Nonspecific antibody binding to the membrane was blocked with $5 \%$ nonfat milk in PBS-Tween $20(0.1 \%, \mathrm{v} / \mathrm{v})$ for 1 hour at room temperature. Membranes were then incubated overnight at $4^{\circ} \mathrm{C}$ with the primary antibodies used against eNOS (1:1,000; BD Biosciences; Oxford, Oxfordshire; UK), phospho-eNOS (1:200; Cell Signaling; Danvers, MA; USA), inducible nitric oxide synthase (iNOS, 1:200; Santacruz; Santacruz, CA; 
UK), Tie-2 (1:2,000; R\&D), phospho-Tie-2 (1:200; R\&D), VE-cadherin (1:200; Cayman; Ann Arbor, MI;USA), phospho-VE-cadherin Y658 (1:1,000; Abcam; Cambridge, Cambridgeshire; UK), Akt (1:5,000; R\&D), phospho-Akt S473 (1:2,000; R\&D), and Ang-2 (1:1,000; Alpha Diagnostic; San Antonio, TX; USA). Detection blots were washed with PBS-Tween $20(0.1 \% \mathrm{v} / \mathrm{v})$ at 10 -minute intervals for 50 minutes and were incubated with horseradish peroxidaseanti-rabbit, horseradish peroxidase-anti-mouse or horseradish peroxidase-anti-goat IgG $(1: 1,000$ to $1: 2,000)$ for 1 hour at room temperature. Primary and secondary antibodies were dissolved in PBS-Tween 20 0.1\% v/v containing 5\% nonfat milk. Membranes were then washed with PBS-Tween $20(0.1 \% \mathrm{v} / \mathrm{v})$ at 10 -minute intervals for 40 minutes and the immunoreactive bands were visualised using an enhanced chemiluminescence system. Every blot was stripped and reprobed for actin (1:1,000; Sigma) as internal control.

\section{Serum nitric oxide assay}

Twenty-four hours after LPS administration, blood serum was obtained by cardiac puncture and centrifuged at 13,000 relative centrifugal force ( $\mathrm{rcf}$ ) for 5 minutes. The Stressgen Nitric Oxide (total) Detection Kit, which quantifies total nitrite colorimetrically using the Griess reaction, was used according to the manufacturer's instructions (Stressgen Biotechnologies; Victoria, British Columbia; Canada). Frozen serum was defrosted, diluted 1:10, and analysed in duplicate. The absorbance in each well between 540 and $570 \mathrm{~nm}$ was determined using a plate reader. The average net absorbance for each standard and sample was calculated by subtracting the average zero standard absorbance from the average absorbance for each standard and sample. This allowed nitrite levels to be calculated as an indicator of total NO production.

\section{Statistical analysis}

All data were expressed as the mean \pm standard error of the mean and analysed using Graphpad Prism version 5.0 (Graphpad Software; San Diego, CA; USA). Two-way analysis of variance followed by the Bonferroni post-hoc test to identify points of significance or one-way analysis of variance followed by appropriate post-hoc test or Student's $t$ test were used as appropriate. $P<0.05$ was considered significant.

\section{Results}

MAT.Ang-1 protects mice against LPS-induced microvascular hyperpermeability

LPS caused a significant increase in macromolecular leak (normalised grey level) compared with controls (LPS group vs. controls, $64.5 \pm 4.9$ vs. $25.0 \pm 6.1$ at 23 hours, $P<0.01 ; 80.7 \pm 10.1$ vs. $35.1 \pm 8.6$ at 24 hours,
$P<0.001)$. MAT.Ang-1 reduced LPS-induced leak at 23 hours $(32.2 \pm 8.3, P<0.05)$ and at 24 hours $(31.6 \pm 9.5$, $P<0.001$ ) (Figure 1). MAT.Ang-1 alone did not significantly increase macromolecular leak compared with controls $(36.5 \pm 5.2$ at 23 hours and $49.7 \pm 7.5$ at 24 hours). However, vessel diameters of arterioles and venules were not significantly different among any experimental group (Table S1 and Figures S1, S2 in Additional file 1).

\section{Microvascular blood flow in sepsis is improved by MAT. Ang-1}

In response to LPS, mean perfusion (percentage of control) decreased to $17.1 \pm 0.6 \%$ at 24 hours $(P<0.01)$ (Figure 2); whereas in the presence of LPS, MAT.Ang-1 increased mean perfusion to $42.1 \pm 11.7 \%(P<0.05)$. However, mean perfusion in response to MAT.Ang-1 alone $(89.0 \pm 18.7 \%)$ was not different from controls.

\section{MAT.Ang-1 reduces the inflammatory response during endotoxemia}

LPS induced a trend of increased expression for the majority of cytokines analysed in skeletal muscle at 24 hours (Table S2 in Additional file 1), including the proinflammatory cytokines IL-1 $\alpha$, IL-1 $\beta$, IL-6, TNF $\alpha$, IFN $\gamma$, soluble intracellular adhesion molecule- 1 (sICAM-1) and triggering receptor expressed on myeloid cells-1 (TREM-1), which were reduced by posttreatment with MAT.Ang-1 (Figure 3). In contrast, monocyte chemotactic protein-1 (MCP-1) and granulocyte colony-stimulating factor (G-CSF) were increased by LPS, but not modulated by MAT.Ang-1. Notably, LPS-induced increased IL-1 $\beta$ expression was significantly reduced by post-treatment with MAT.Ang-1 $(P<0.05)$ (Figure 3). MAT.Ang-1 alone also reduced the expression of TNF $\alpha$, IFN $\gamma$, triggering receptor expressed on myeloid cells- 1 and G-CSF $(P<0.05)$. Furthermore, the anti-inflammatory cytokines IL-10 and IL-1 receptor antagonist exhibited increased expression during endotoxemia, but were reduced by post-treatment with MAT.Ang-1. IL-10 was also reduced by MAT-Ang-1 alone $(P<0.01)$ (Figure 3 ).

\section{Angiogenic markers are reduced in sepsis and not induced by MAT.Ang-1}

The majority of angiogenic factors were significantly reduced at 24 hours in response to LPS (Table S3 in Additional File 1). In particular, Ang-1 and Ang-3 $(P<$ $0.001)$, endothelin-1 $(P<0.01)$ and tissue factor $(P<$ 0.01 ) were significantly reduced (Figure 4$)$. Post-treatment with MAT.Ang-1 during endotoxemia did not increase significantly the expression of Ang-1, Ang-3, endothelin-1 and tissue factor. In addition, vascular endothelial growth factor (VEGF) expression was 


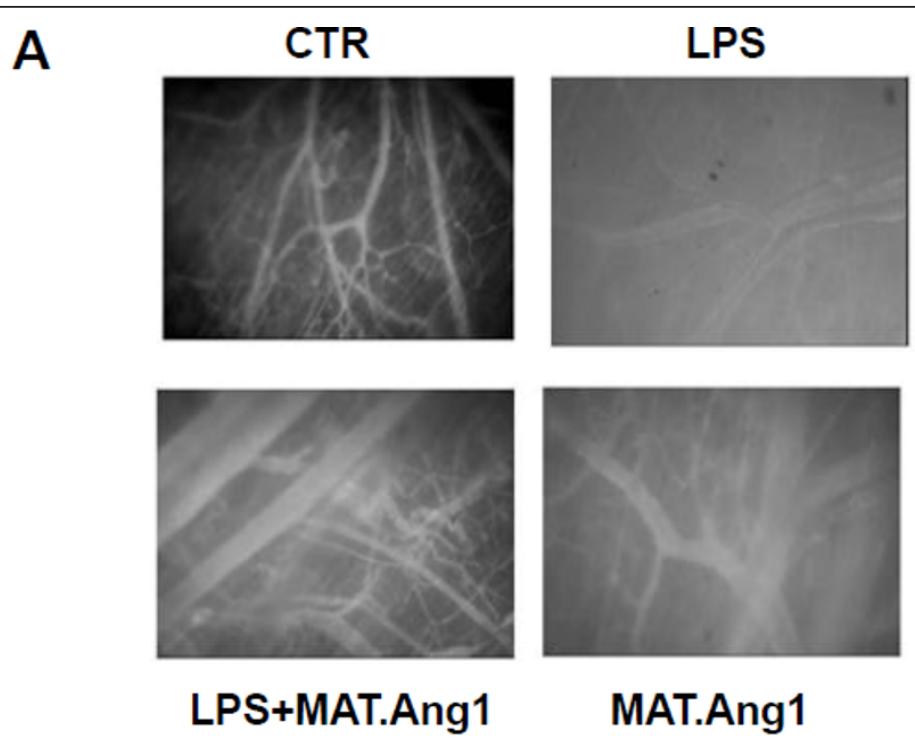

B

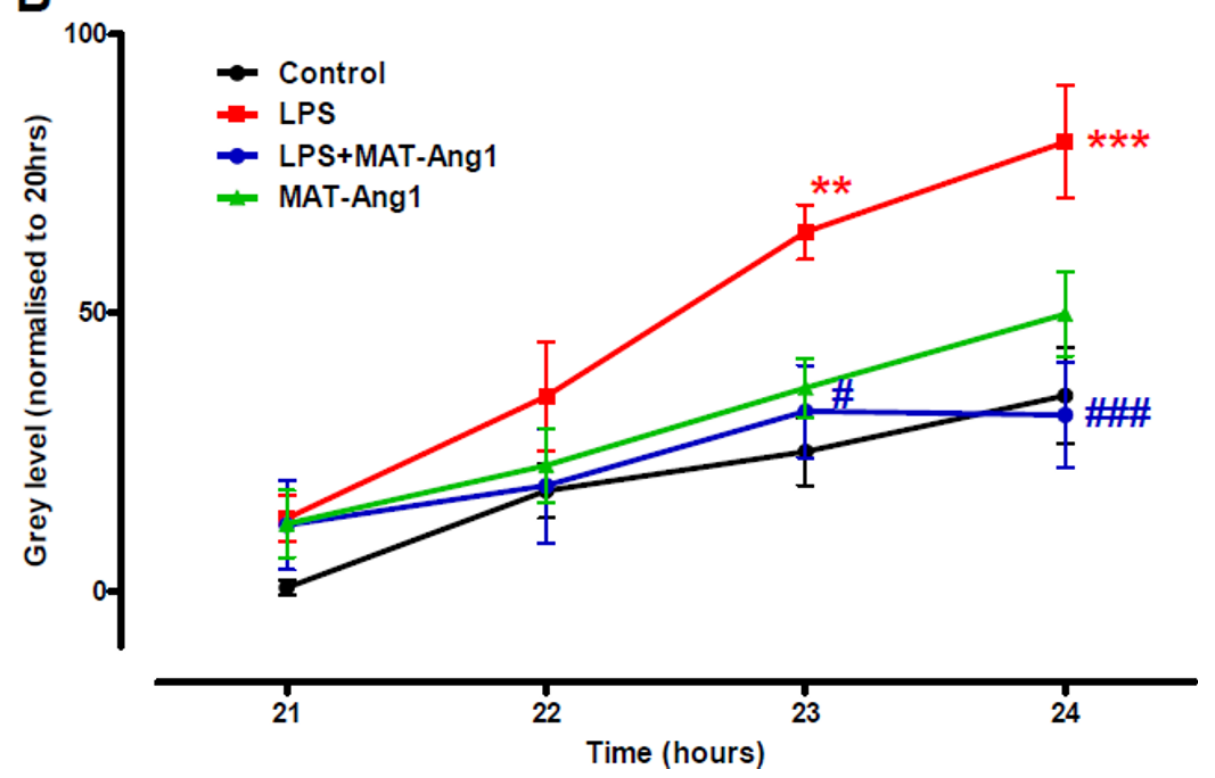

Figure 1 MAT.Ang-1 reduces lipopolysaccharide-induced microvascular leak. (A) Representative images at 24 hours of murine skeletal muscle microvasculature from control (CTR), lipopolysaccharide (LPS)-treated, LPS + matrilin-1-angiopoietin-1 (MAT-Ang1)-treated and MAT-Ang1treated groups. (B) Data expressed as mean \pm standard error of the mean for change in grey level indicating macromolecular leak from 20 hours $(n=6) .{ }^{*} P<0.01$ and ${ }^{* * *} P<0.001$ vs. control. ${ }^{\#} P<0.05$ and ${ }^{\# \# \#} P<0.001$ vs. LPS.

reduced in the presence of MAT.Ang-1 alone $(P<0.05)$ and during endotoxemia $(P<0.01)$.

Ang-2 expression was significantly increased in endotoxemia at 24 hours $(P<0.01)$, and was unchanged by MAT.Ang-1 administration (Figure 5). Conversely, Tie-2 receptor expression was significantly reduced by LPS at 24 hours $(P<0.05)$, and was unchanged by MAT.Ang-1 (Figure 5). Furthermore, Tie-2 phosphorylation at Y1100 by MAT.Ang-1 was observed in normality but not in response to LPS (Figure 5). At the same time point, the signalling survival protein Akt and its phosphorylation at S473 were not significantly different amongst the experimental groups (Figure 5).

\section{MAT.Ang-1 increases VE-cadherin phosphorylation in sepsis}

No change in VE-cadherin expression was observed amongst the experimental groups (Figure 6). Nevertheless, during endotoxemia VE-cadherin phosphorylation at Y658 was increased in MAT.Ang-1-treated animals compared 

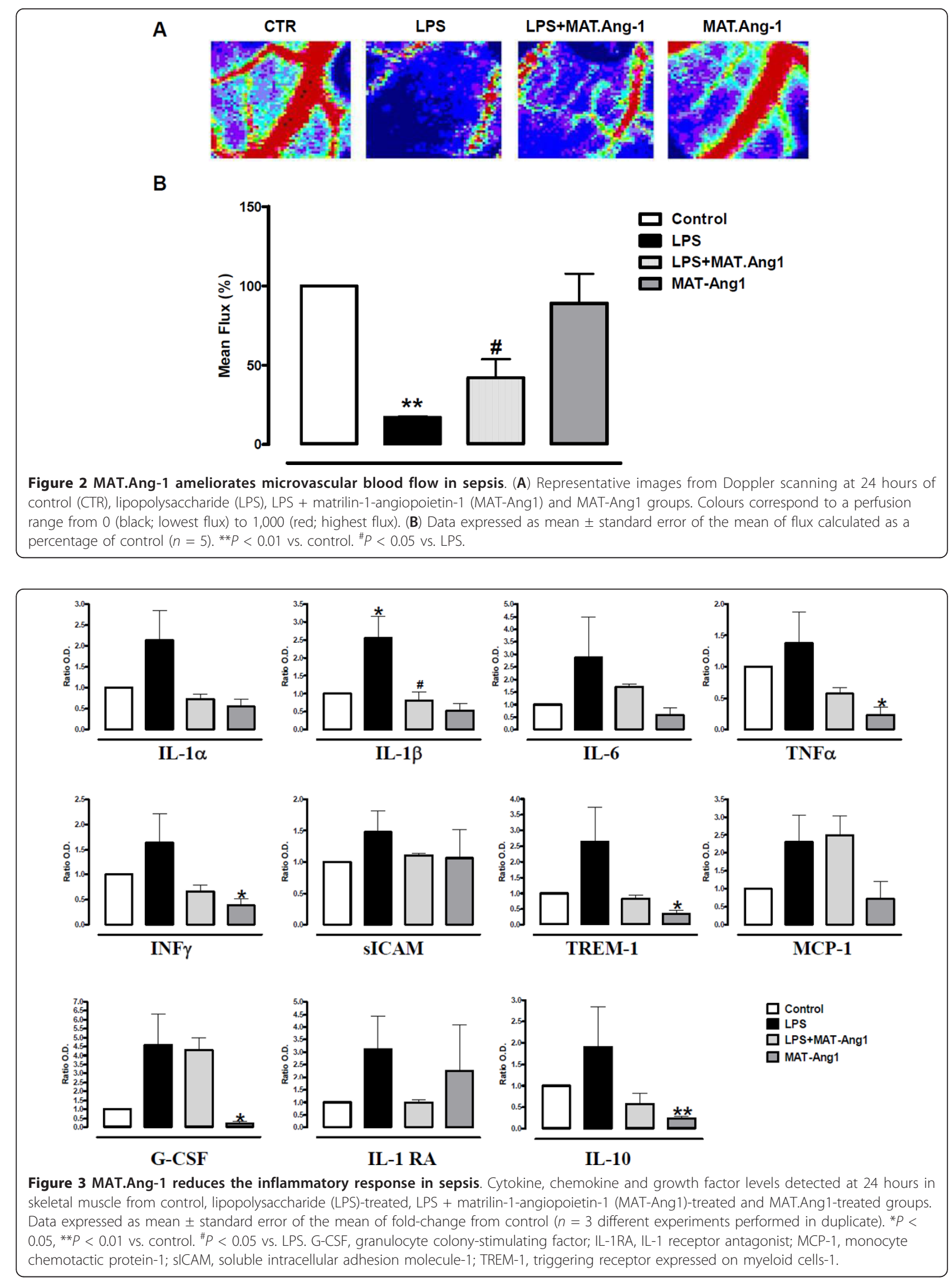


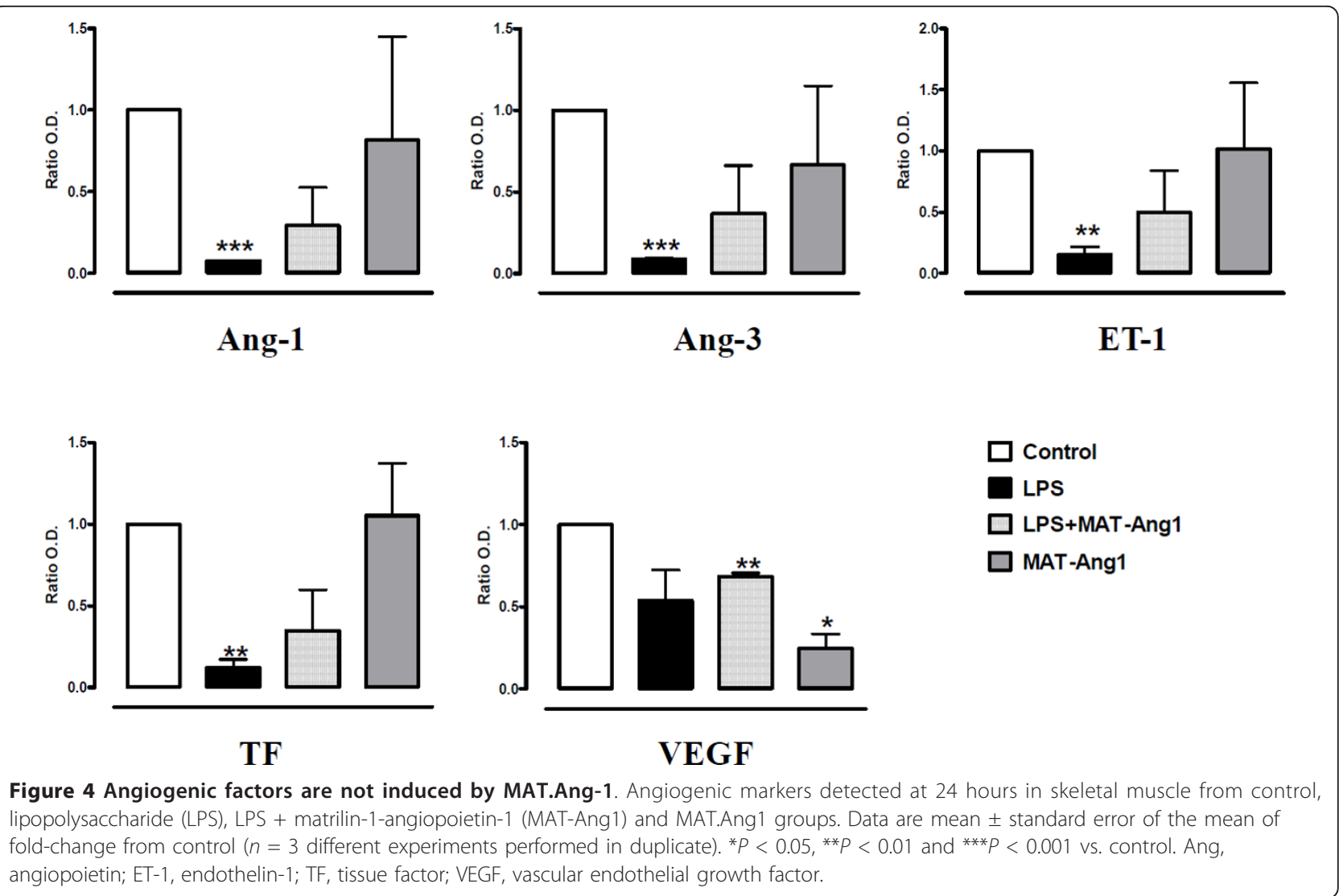

with those treated with LPS alone $(P<0.05)$ (Figure 6). MAT.Ang-1 alone also reduced Y658 phosphorylation, but this did not reach significance $(P=0.07)$.

\section{LPS-induced increase in nitric oxide synthase expression is not changed by MAT.Ang-1}

eNOS phosphorylation at Ser1177 did not vary in skeletal muscle amongst the experimental groups (Figure 6). Nevertheless, eNOS protein expression increased after 24 hours of endotoxemia and this was not altered by post-treatment with MAT.Ang- 1 ( $P<0.05$ vs. control) (Figure 6). iNOS expression was neither detected in controls nor in the presence of MAT.Ang-1 alone, and the LPS-induced increase in iNOS expression $(P<0.01)$ was unchanged by MAT.Ang-1 (Figure 6).

Serum NO, measured as total nitrite levels, increased from $53.2 \pm 26.6 \mu \mathrm{mol} / \mathrm{l}$ in controls to $342.1 \pm 118.7$ $\mu \mathrm{mol} / \mathrm{l}$ in endotoxemic mice $(P<0.05)$, and remained elevated with concurrent LPS and MAT.Ang-1 administration $(551.2 \pm 245.3 \mu \mathrm{mol} / \mathrm{l})$. NO levels were also similar to controls with MAT.Ang-1 alone $(52.7 \pm 44.8 \mu \mathrm{mol} / \mathrm{l})$.

\section{Discussion}

This is the first in vivo demonstration that MAT.Ang-1, administered after the onset of sub-lethal endotoxemia, reduces LPS-induced macromolecular leak and improves microcirculatory blood flow in sepsis without changing vascular resistance. In contrast, MAT.Ang-1 alone does not induce leak or change microvascular flow and/or resistance in skeletal muscle under physiological conditions. Endotoxemia is locally associated with increased inflammation and reduced angiogenic factors. MAT. Ang-1 post-treatment reduces the majority of inflammatory mediators, including IL-1 $\beta$, without increasing the expression of angiogenic factors. Under physiological conditions, MAT.Ang-1 does not induce local angiogenic markers, but reduces VEGF expression. Our data therefore show that MAT.Ang-1 protects the microcirculation from sepsis-induced vascular dysfunction and local inflammation without causing angiogenesis.

In search of an efficacious treatment for sepsis our study has focused on the microcirculation, based on recent evidence that microcirculatory dysfunction is a major feature of sepsis [2]. Microvascular dysfunction in sepsis is characterised by disruption of endothelial barrier function [21], but no effective pharmacological therapy is currently available to reduce the increased vascular permeability occurring in sepsis [22]. In our study, MAT. Ang-1 administered following the onset of sepsis protected the microcirculation against the barrier breakdown 


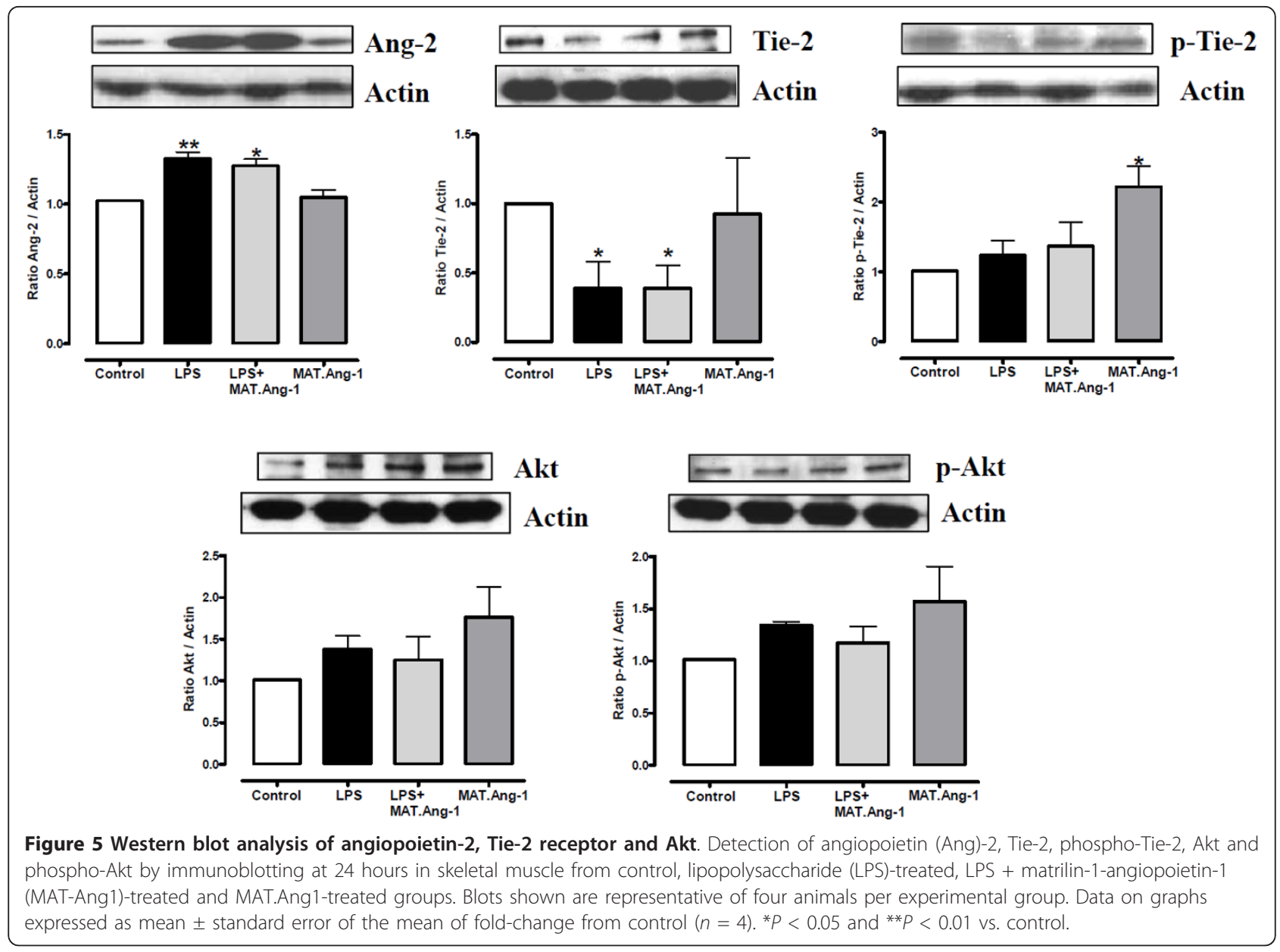

induced by experimental endotoxemia. Consistently, microcirculatory perfusion was found to be dramatically reduced in endotoxemic mice and improved following MAT.Ang-1 administration. Moreover, we applied the laser Doppler imaging technique to the window chamber model for the first time, and propose that the intravital microscopy-Doppler combination is a powerful technology to study the microcirculation in health and disease. Notably, MAT.Ang-1 did not change the arteriolar diameter during sepsis and normal conditions, suggesting that: the effects of MAT.Ang-1 on microvascular permeability and blood flow in pathological (endotoxemia) and physiological conditions are not dependent on changes in microvascular resistance; and MAT.Ang-1-induced recovery of microcirculatory tissue perfusion during sepsis is due to preservation of endothelial barrier integrity.

Endothelial cell damage and inflammation have been considered the basis of increased vascular permeability and altered blood flow [23]. We used a protein antibody array for the simultaneous detection of 40 different cytokines, chemokines and growth factors to investigate whether MAT.Ang-1 protected the microcirculation by modulating the inflammatory response occurring in sepsis. Most inflammatory cytokines showed a trend of increased expression in endotoxemia and reduction in the presence of MAT.Ang-1. Notably, the reduced expression of IL- $1 \beta$ by MAT.Ang- 1 may account for the beneficial action on blood flow and tissue perfusion in endotoxemia, as IL-1 $\beta$ induces neutrophil migration into tissue, causing microvascular stasis in sepsis $[24,25]$. Furthermore, TNF $\alpha$ and IL- 6 are considered biomarkers of sepsis [26,27], and in this study their levels were reduced locally by MAT.Ang-1 during endotoxemia. By contrast, G-CSF and monocyte chemotactic protein-1 expression remained elevated after MAT.Ang- 1 administration in sepsis. G-CSF has been included in clinical trials for the prophylaxis and treatment of sepsis $[28,29]$, while monocyte chemotactic protein-1 may be involved in the pathophysiology of sepsis as plasma levels of this chemokine are elevated in septic patients [30], but its role remains controversial $[31,32]$.

The role of angiogenic factors in the pathogenesis of sepsis is largely unexplored, but therapeutic induction of angiogenesis in damaged vessels has been suggested as a 


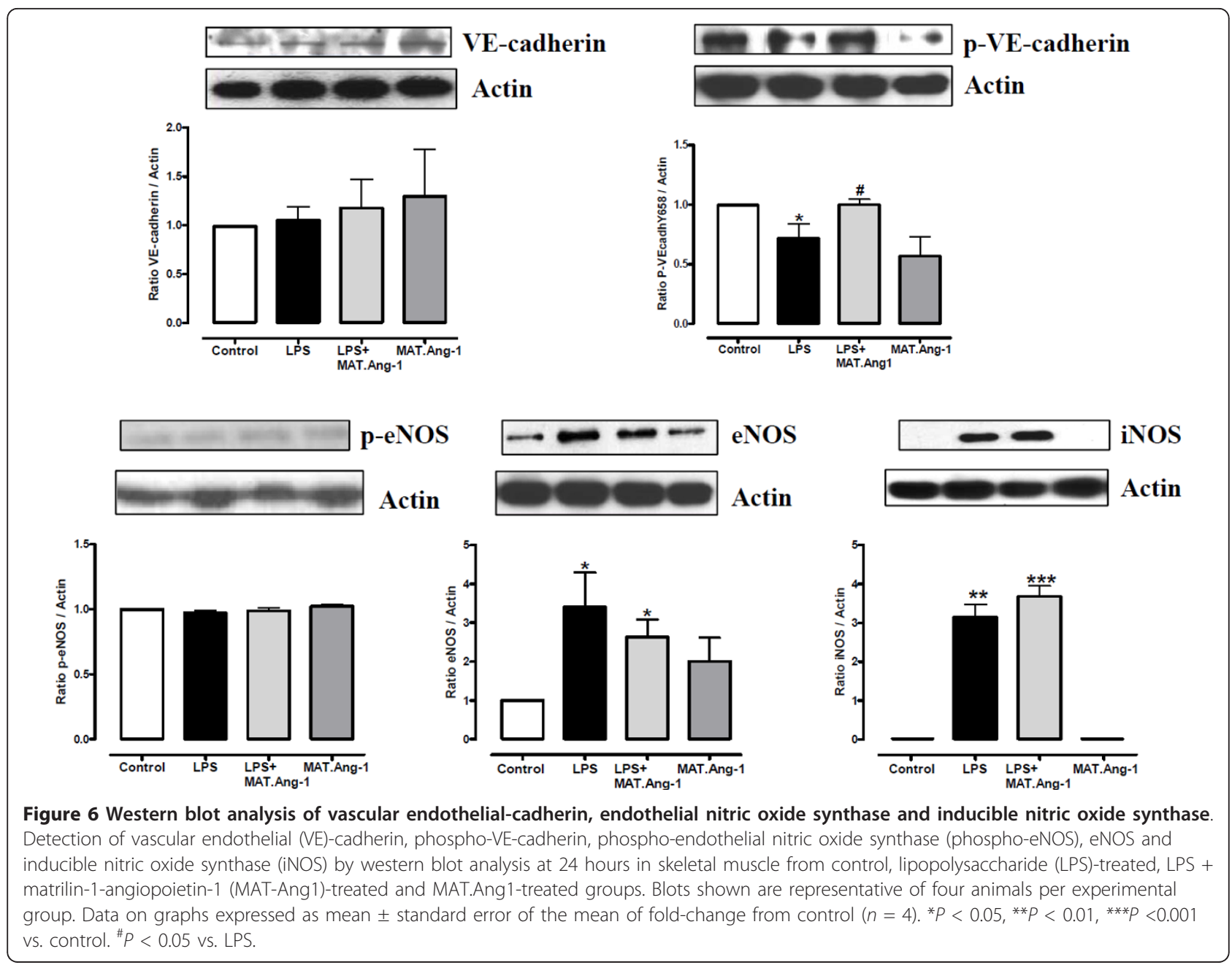

valid approach $[33,34]$. Our results demonstrate a significant reduction during endotoxemia in local angiogenic factors, including Ang-1, Ang-3, endothelin-1 and tissue factor, with no marked changes by MAT.Ang-1. Consistently, we observed no change in expression and phosphorylation of the pro-survival factor Akt or eNOS phosphorylation at Ser1177 at the same time point. The absence of pro-angiogenic effects by MAT.Ang-1 is unsurprising because Ang-1 regulates vascular maturation at later stages of the angiogenic cascade [8]. Moreover, our findings show that LPS increased Ang-2 protein levels with concomitant reduction in Tie-2 receptor expression in skeletal muscle, thus providing further in vivo evidence that endotoxemia triggers inhibition of the Ang/Tie system, which may contribute to vascular dysfunction in sepsis [35]. Furthermore, our results suggest that MAT.Ang- 1 acts via Tie- 2 in vivo with increased receptor phosphorylation, although specific receptor activation by MAT.Ang-1 in vivo is difficult to verify because concomitant receptor blockade - for example, by Ang-2 in inflammation - modulates vascular function (see also Figure S3 in Additional file 1). However, Tie-2phosphorylation was unchanged during endotoxemia.

Previous studies demonstrated that VE-cadherin phosphorylation at the critical tyrosine Y658 may be sufficient to inhibit cell barrier function [36]. Nevertheless, disassembly of VE-cadherin junctions has been shown to trigger an intracellular negative signal for limiting transendothelial leukocyte migration in mice 6 hours after challenge with LPS [37]. Increased phosphorylation at Y658 in endotoxemia by MAT.Ang-1, in addition to reduced IL- $1 \beta$ expression, is therefore likely to reduce neutrophil infiltration and microvascular stasis in sepsis.

During the progression of sepsis, proinflammatory cytokines and LPS stimulate NO production mainly through increased expression of iNOS, which has been implicated in the pathophysiology of microcirculatory failure and organ dysfunction [38]. However, in the present study neither iNOS nor total nitrite levels were modulated by the administration of MAT.Ang-1, despite the observed beneficial effects on reducing inflammation. 
Moreover, MAT.Ang-1 also sustained the increase in tissue eNOS expression during endotoxemia, which may act as a protective mechanism in sepsis by improving microcirculatory flow [39]. Recent evidence also suggests that high levels of NO can be beneficial in sepsis by improving tissue perfusion and oxygen extraction [40], thus NO is part of a complex mechanistic pathway and further studies are required before firm conclusions can be made regarding an inflammatory role for NO [41].

Previous studies have used COMP-Ang-1 prophylactically to prevent vascular inflammation and organ dysfunction during sepsis [42,43], and our findings demonstrate that MAT.Ang-1 has similar protective effects even administered after the onset of endotoxemia. Since MAT.Ang-1 and Ang-1 have similar physiological activity, this suggests that our novel variant is likely to demonstrate increased therapeutic potential when compared with COMP.Ang-1 [13], although this requires confirmation. Biological MAT.Ang-1 units consist of trimers and tetramers rather than more active pentamers, and hence - compared with COMP.Ang-1 side effects such as venous malformations are less likely to occur when administered systemically [44]. Moreover, while previous studies have shown protective effects by repeated prophylactic or therapeutic injections of recombinant Ang-1 [45] or the Tie-2 agonist Vasculotide [46] in murine sepsis, the current study demonstrates that a single administration of the stable variant MAT.Ang-1 after the onset of sepsis improved endotoxemia-induced microvascular dysfunction.

Using the variant MAT.Ang-1, this in vivo study also further investigated the effects of acute activation of the angiopoietin system under physiological conditions. In skeletal muscle, TNF $\alpha$, IFN $\gamma$, IL-10 and G-CSF expression were reduced by MAT.Ang-1, further confirming that Ang-1 may act as an endogenous vessel stabiliser [47]. By contrast, MAT.Ang-1 did not alter the expression of any angiogenic factors detected, suggesting that induction of angiogenesis is unlikely at the dose used. Interestingly, only VEGF expression was reduced by MAT.Ang-1 administration. Indeed, a functional competition has previously been described between VEGF and Ang-1, with Ang-1 preventing vascular leakage in response to VEGF administration [48]. VEGF also induces cleavage of Tie-2 receptors, creating soluble forms (soluble Tie-2) that sequester Ang-1 and inhibit ligand-mediated Tie-2 activation [49]. Our data suggest for the first time that a feedback mechanism exists by which Ang-1 downregulates VEGF-mediated vascular instability, resulting in vascular stabilisation.

\section{Conclusion}

Our study provides evidence that the microcirculation is protected from sepsis-induced vascular dysfunction in vivo by acute parenteral administration of MAT.Ang-1, a novel variant of Ang-1, after the onset of endotoxemia. To our knowledge, no other studies have successfully used an angiopoietin variant to reverse inflammation - and our data show that vascular protection is mediated by effects on the inflammatory response in sepsis rather than stimulation of angiogenesis. Further studies are also warranted to examine the ability of MAT.Ang- 1 to reduce mortality in more severe models of sepsis. Pharmacotherapeutic targeting of the angiopoietin system in sepsis, in addition to other cardiovascular disorders, is thus possible using chemical variants of Ang- 1 that improve its pharmacokinetic and pharmacodynamic characteristics in vivo, allowing optimal delivery [50]. Improved microvascular blood flow, reduced inflammation and lack of activation of angiogensis by MAT.Ang-1 post-treatment in our nonlethal in vivo model of sepsis provide the basis for further clinical investigation with septic patients.

\section{Key messages}

- Ang-1 showed therapeutic potential for the treatment of sepsis and cardiovascular disorders, but further pharmacological characterisation and drug delivery is required. This study aimed to investigate the acute effects of the stable and active Ang-1 variant MAT.Ang- 1 on the microcirculatory function (permeability, resistance and blood flow) in an experimental model of sepsis.

- Endotoxemia resulted in microvascular leak and reduction of tissue perfusion, which was ameliorated by MAT.Ang-1 post-treatment.

- MAT.Ang-1 post-treatment reduced the inflammatory response by LPS but did not significantly induce the expression of angiogenic factors.

- MAT.Ang-1 alone did not induce leak or increase angiogenic factors but, in contrast, reduced VEGF expression in controls.

- Administration of MAT.Ang-1 after the onset of sepsis protects the microcirculation from endotoxemia-induced vascular dysfunction by reducing the inflammatory response but with no concomitant induction of angiogenic factors, thus representing a novel drug for the treatment of sepsis.

\section{Additional material}

Additional file 1: Table S1 presenting vessel diameters from control, LPS, LPS+MAT-Ang1 and MAT-Ang1 groups. Table S2 presenting protein expression of cytokines and chemokines in skeletal muscle from control, LPS, LPS+MAT-Ang1 and MAT-Ang1 groups. Table S3 presenting protein expression of angiogenic factors in skeletal muscle from control, LPS, LPS+MAT-Ang1 and MAT-Ang1 groups. Figure S1 showing diameters of primary arterioles and venules from control, LPS, LPS+MATAng1 and MAT-Ang1 groups. Figure S2 showing diameters of precapillary arterioles and post-capillary venules from control, LPS, LPS+MAT- 
Ang1 and MAT-Ang1 groups. Figure S3 showing the effect of Tie-2 receptor antagonist NLLMAAS on macromolecular leak.

\section{Abbreviations}

Akt: RAC - a serine/threonine-protein kinase; Ang: angiopoietin; BSA: bovine serum albumin; eNOS: endothelial nitric oxide synthase; G-CSF: granulocyte colony-stimulating factor; IL: interleukin; iNOS: inducible nitric oxide synthase; i.p.: intraperitoneally; LPS: lipopolysaccharide; MAT.Ang-1: matrilin-1angiopoietin-1; mDia: mammalian diaphanous; NO: nitric oxide; PBS: phosphate-buffered saline; PCR: polymerase chain reaction; Tie-2: tyrosine kinase with immunoglobulin-like and endothelial growth factor-like domains 2; VE: vascular endothelial; VEGF: vascular endothelial growth factor.

\section{Acknowledgements}

The authors wish to thank Dr Sheila Francis for her kind support with the laser Doppler imaging and Dr Robert Skinner for his assistance with establishing the dorsal microcirculatory chamber model in sepsis (University of Sheffield). This study was supported by the British Heart Foundation (Project Grant No. 06/080).

\section{Author details}

'Microcirculation Research Group, Faculty of Medicine, Dentistry and Health, University of Sheffield, Sheffield S10 2RX, UK. ${ }^{2}$ Laboratory of Biomolecular Research, Paul Scherrer Institut, CH-5232 Villigen PSI, Switzerland. " Wellcome Trust Centre for Cell-Matrix Research, Faculty of Life Sciences, University of Manchester, Manchester M13 9PT, UK.

\section{Authors' contributions}

All in vivo and ex vivo experiments in this study were performed by AA. MAT.Ang-1 was produced by MT and PP in collaboration with RAK. JJW performed preliminary experiments in collaboration with KR. ZLB and NJB had the initial idea, obtained the funding, led and supervised the project. The manuscript was written by AA and ZLB in consultation with NJB and RAK, who contributed to aspects of data analysis. All authors read and approved the final version of this manuscript.

\section{Competing interests}

The authors declare that they have no competing interests.

Received: 15 May 2012 Revised: 14 August 2012

Accepted: 4 October 2012 Published: 4 October 2012

\section{References}

1. Hawiger J, Musser JM: How to approach gene wars in sepsis? Crit Care 2011, 15:1007.

2. Spronk PE, Zandstra DF, Ince C: Bench-to-bedside review: sepsis is a disease of the microcirculation. Crit Care 2004, 8:462-468.

3. Ince C: The microcirculation is the motor of sepsis. Crit Care 2005, 9(Suppl 4):S13-S19.

4. Jones AE, Puskarich MA: Sepsis-induced tissue hypoperfusion. Crit Care Clin 2009, 25:769-779, ix.

5. Angus DC, Linde-Zwirble WT, Lidicker J, Clermont G, Carcillo J, Pinsky MR: Epidemiology of severe sepsis in the United States: analysis of incidence, outcome, and associated costs of care. Crit Care Med 2001, 29:1303-1310.

6. Dellinger RP, Levy MM, Carlet JM, Bion J, Parker MM, Jaeschke R, Reinhart K, Angus DC, Brun-Buisson C, Beale R, Calandra T, Dhainaut JF, Gerlach H, Harvey M, Marini JJ, Marshall J, Ranieri M, Ramsay G, Sevransky J, Thompson BT, Townsend S, Vender JS, Zimmerman JL, Vincent JL: Surviving Sepsis Campaign: international guidelines for management of severe sepsis and septic shock: 2008. Crit Care Med 2008, 36:296-327.

7. Brindle NP, Saharinen P, Alitalo K: Signaling and functions of angiopoietin1 in vascular protection. Circ Res 2006, 98:1014-1023.

8. Augustin $\mathrm{HG}$, Koh GY, Thurston G, Alitalo K: Control of vascular morphogenesis and homeostasis through the angiopoietin-Tie system. Nat Rev Mol Cell Biol 2009, 10:165-177.
9. Partovian C, Zhuang Z, Moodie K, Lin M, Ouchi N, Sessa WC, Walsh K, Simons M: PKCa activates eNOS and increases arterial blood flow in vivo. Circ Res 2005, 97:482-487.

10. Witzenbichler B, Westermann D, Knueppel S, Schultheiss HP, Tschope C: Protective role of angiopoietin-1 in endotoxic shock. Circulation 2005, 111:97-105.

11. Mammoto T, Parikh SM, Mammoto A, Gallagher D, Chan B, Mostoslavsky G, Ingber DE, Sukhatme VP: Angiopoietin-1 requires p190 RhoGAP to protect against vascular leakage in vivo. J Biol Chem 2007, 282:23910-23918.

12. Zhu YG, Qu JM, Zhang J, Jiang HN, Xu JF: Novel interventional approaches for ALI/ARDS: cell-based gene therapy. Mediators Inflamm 2011, 2011:560194.

13. Cho CH, Kammerer RA, Lee HJ, Steinmetz MO, Ryu YS, Lee SH, Yasunaga $K$, Kim KT, Kim I, Choi HH, Kim W, Kim SH, Park SK, Lee GM, Koh GY: COMPAng1: a designed angiopoietin-1 variant with nonleaky angiogenic activity. Proc Natl Acad Sci USA 2004, 101:5547-5552.

14. Macdonald PR, Progias P, Ciani B, Patel S, Mayer U, Steinmetz MO, Kammerer RA: Structure of the extracellular domain of Tie receptor tyrosine kinases and localization of the angiopoietin-binding epitope. J Biol Chem 2006, 281:28408-28414.

15. Poschl E, Fox JW, Block D, Mayer U, Timpl R: Two non-contiguous regions contribute to nidogen binding to a single EGF-like motif of the laminin gamma 1 chain. EMBO J 1994, 13:3741-3747.

16. Bingle L, Lewis CE, Corke KP, Reed MW, Brown NJ: Macrophages promote angiogenesis in human breast tumour spheroids in vivo. Br J Cancer 2006, 94:101-107.

17. Brookes ZL, Stedman EN, Guerrini R, Lawton BK, Calo G, Lambert DG: Proinflammatory and vasodilator effects of nociceptin/orphanin FQ in the rat mesenteric microcirculation are mediated by histamine. Am J Physiol Heart Circ Physiol 2007, 293:H2977-H2985.

18. Cho CH, Kammerer RA, Lee HJ, Yasunaga K, Kim KT, Choi HH, Kim W, Kim SH, Park SK, Lee GM, Koh GY: Designed angiopoietin-1 variant, COMP-Ang1, protects against radiation-induced endothelial cell apoptosis. Proc Natl Acad Sci USA 2004, 101:5553-5558.

19. Allcock GH, Allegra M, Flower RJ, Perretti M: Neutrophil accumulation induced by bacterial lipopolysaccharide: effects of dexamethasone and annexin 1. Clin Exp Immunol 2001, 123:62-67.

20. McGown CC, Brown NJ, Hellewell PG, Reilly CS, Brookes ZL: Beneficial microvascular and anti-inflammatory effects of pravastatin during sepsis involve nitric oxide synthase III. Br J Anaesth 2010, 104:183-190.

21. Matsuda N, Hattori Y: Vascular biology in sepsis: pathophysiological and therapeutic significance of vascular dysfunction. J Smooth Muscle Res 2007, 43:117-137.

22. Lee WL, Liles WC: Endothelial activation, dysfunction and permeability during severe infections. Curr Opin Hematol 2011, 18:191-196.

23. Aird WC: The role of the endothelium in severe sepsis and multiple organ dysfunction syndrome. Blood 2003, 101:3765-3777.

24. Pettipher ER, Higgs GA, Henderson B: Interleukin 1 induces leukocyte infiltration and cartilage proteoglycan degradation in the synovial joint. Proc Natl Acad Sci USA 1986, 83:8749-8753.

25. Rampart M, Williams TJ: Evidence that neutrophil accumulation induced by interleukin-1 requires both local protein biosynthesis and neutrophil CD18 antigen expression in vivo. Br J Pharmacol 1988, 94:1143-1148.

26. Bateman RM, Sharpe MD, Ellis CG: Bench-to-bedside review: microvascular dysfunction in sepsis - hemodynamics, oxygen transport, and nitric oxide. Crit Care 2003, 7:359-373.

27. Damas $P$, Canivet $J$, de GD, Vrindts $Y$, Albert A, Franchimont P, Lamy M: Sepsis and serum cytokine concentrations. Crit Care Med 1997, 25:405-412.

28. Pinsky MR, Vincent JL, Deviere J, Alegre M, Kahn RJ, Dupont E: Serum cytokine levels in human septic shock. Relation to multiple-system organ failure and mortality. Chest 1993, 103:565-575.

29. Murata A: Granulocyte colony-stimulating factor as the expecting sword for the treatment of severe sepsis. Curr Pharm Des 2003, 9:1115-1120.

30. Pugin J: Immunostimulation is a rational therapeutic strategy in sepsis. Novartis Found Symp 2007, 280:21-27.

31. Bossink AW, Paemen $L$, Jansen PM, Hack CE, Thijs LG, Van DJ: Plasma levels of the chemokines monocyte chemotactic proteins- 1 and -2 are elevated in human sepsis. Blood 1995, 86:3841-3847. 
32. Zisman DA, Kunkel SL, Strieter RM, Tsai WC, Bucknell K, Wilkowski J, Standiford TJ: MCP-1 protects mice in lethal endotoxemia. J Clin Invest 1997, 99:2832-2836.

33. Al SH: Therapeutic angiogenesis in cardiovascular disease. J Cardiothorac Surg 2007, 2:49.

34. Mankhambo LA, Banda DL, Jeffers $G$, White $S A$, Balmer P, Nkhoma $S$, Phiri H, Molyneux EM, Hart CA, Molyneux ME, Heyderman RS, Carrol ED: The role of angiogenic factors in predicting clinical outcome in severe bacterial infection in Malawian children. Crit Care 2010, 14:R91.

35. Mofarrahi M, Nouh T, Qureshi S, Guillot L, Mayaki D, Hussain SN: Regulation of angiopoietin expression by bacterial lipopolysaccharide. Am J Physiol Lung Cell Mol Physiol 2008, 294:L955-L963.

36. Potter MD, Barbero S, Cheresh DA: Tyrosine phosphorylation of VEcadherin prevents binding of $\mathrm{p} 120$ - and beta-catenin and maintains the cellular mesenchymal state. J Biol Chem 2005, 280:31906-31912.

37. Orrington-Myers J, Gao X, Kouklis P, Broman M, Rahman A, Vogel SM, Malik AB: Regulation of lung neutrophil recruitment by VE-cadherin. Am J Physiol Lung Cell Mol Physiol 2006, 291:L764-L771.

38. Cunha FQ, Assreuy J, Moss DW, Rees D, Leal LM, Moncada S, Carrier M, O'Donnell CA, Liew FY: Differential induction of nitric oxide synthase in various organs of the mouse during endotoxaemia: role of TNF-alpha and IL-1-beta. Immunology 1994, 81:211-215.

39. Hollenberg SM, Cinel I: Bench-to-bedside review: nitric oxide in critical illness - update 2008. Crit Care 2009, 13:218.

40. Buwalda M, Ince C: Opening the microcirculation: can vasodilators be useful in sepsis? Intensive Care Med 2002, 28:1208-1217.

41. Pacher P, Beckman JS, Liaudet L: Nitric oxide and peroxynitrite in health and disease. Physiol Rev 2007, 87:315-424.

42. Hwang JA, Lee EH, Lee SD, Park JB, Jeon BH, Cho CH: COMP-Ang1 ameliorates leukocyte adhesion and reinforces endothelial tight junctions during endotoxemia. Biochem Biophys Res Commun 2009, 381:592-596.

43. Kim DH, Jung YJ, Lee AS, Lee S, Kang KP, Lee TH, Lee SY, Jang KY, Moon WS, Choi KS, Yoon KH, Sung MJ, Park SK, Kim W: COMPangiopoietin-1 decreases lipopolysaccharide-induced acute kidney injury. Kidney Int 2009, 76:1180-1191.

44. Eklund L, Olsen BR: Tie receptors and their angiopoietin ligands are context-dependent regulators of vascular remodeling. Exp Cell Res 2006, 312:630-641

45. David S, Park JK, Meurs M, Zijlstra JG, Koenecke C, Schrimpf C, Shushakova N, Gueler F, Haller H, Kumpers P: Acute administration of recombinant angiopoietin-1 ameliorates multiple-organ dysfunction syndrome and improves survival in murine sepsis. Cytokine 2011, 55:251-259.

46. Kumpers P, Gueler F, David S, Van SP, Dumont DJ, Park JK, Bockmeyer CL, Parikh SM, Pavenstadt H, Haller H, Shushakova N: The synthetic Tie2 agonist peptide vasculotide protects against vascular leakage and reduces mortality in murine abdominal sepsis. Crit Care 2011, 15:R261.

47. Thomas M, Augustin HG: The role of the Angiopoietins in vascular morphogenesis. Angiogenesis 2009, 12:125-137.

48. Vestweber D: VE-cadherin: the major endothelial adhesion molecule controlling cellular junctions and blood vessel formation. Arterioscler Thromb Vasc Biol 2008, 28:223-232.

49. Findley CM, Cudmore MJ, Ahmed A, Kontos CD: VEGF induces Tie2 shedding via a phosphoinositide 3-kinase/Akt dependent pathway to modulate Tie2 signaling. Arterioscler Thromb Vasc Biol 2007, 27:2619-2626.

50. Novotny NM, Lahm T, Markel TA, Crisostomo PR, Wang M, Wang Y, Tan J, Meldrum DR: Angiopoietin-1 in the treatment of ischemia and sepsis. Shock 2009, 31:335-341.

doi: $10.1186 / \mathrm{cc} 11666$

Cite this article as: Alfieri et al: Angiopoietin-1 variant reduces LPSinduced microvascular dysfunction in a murine model of sepsis. Critical Care 2012 16:R182.

\section{Submit your next manuscript to BioMed Central and take full advantage of:}

- Convenient online submission

- Thorough peer review

- No space constraints or color figure charges

- Immediate publication on acceptance

- Inclusion in PubMed, CAS, Scopus and Google Scholar

- Research which is freely available for redistribution 